\title{
EFEITOS DA COMUNIDADE DE ORIGEM NO PARTICIPANTE DE COMUNIDADES VIRTUAIS DE MARCA
}

\author{
COMMUNITY OF ORIGIN EFFECTS ON VIRTUAL BRAND COMMUNITIES PARTICIPANT \\ EFECTOS DE LA COMUNIDAD DE ORIGEN EN EL PARTICIPANTE DE COMUNIDADES VIRTUALES DE MARCA
}

\section{RESUMO}

Os benefícios das empresas em manterem comunidades virtuais de marca próprias versus os provenientes das comunidades criadas pelos consumidores permanecem desconhecidos. Esse estudo testa o efeito moderador do tipo de gestão da comunidade no impacto da homogeneidade psicográfica percebida, da disponibilidade de avenidas virtuais e do relacionamento com a marca sobre as influências da comunidade e as intenções dos participantes da comunidade. Os dados provêm de 555 participantes ativos de duas grandes comunidades virtuais para o game XBOX no Brasil. Os resultados comprovam que a gestão da comunidade de origem é moderadora de todas as relações consideradas. Apontam, também, que alguns dos efeitos mais favoráveis para a empresa ocorrem na comunidade gerida pelos consumidores. A lealdade à marca, porém, é maior para membros da comunidade oficial. O estudo indica como as empresas podem se beneficiar de comunidades geridas por consumidores.

PALAVRAS-CHAVE Comportamento do consumidor, comunidades virtuais, comunidades de marca, marca, estratégia de marketing.

Stefânia Ordovás de Almeida stefania.almeida@terra.com.br

Professora do Programa de Pós-Graduação em Administração, Universidade Católica do Rio Grande do Sul - Porto Alegre - RS, Brasil

José Afonso Mazzon jamazzon@usp.br

Professor da Faculdade de Economia Administração e Contabilidade, Departamento de Administração, Universidade de São

Paulo - São Paulo - SP, Brasil

Hugo Fridolino Müller Neto hmullerneto@gmail.com

Professor da Escola de Administração, Universidade Federal do Rio Grande do Sul - Porto Alegre - RS, Brasil

Utpal Dholakia dholakia@rice.edu

Professor da Jones Graduate School of Business, Rice University - Houston - TX, Estados Unidos

\begin{abstract}
The question about the effectiveness of companies in maintaining their own communities versus benefiting from the ones owned by consumers remains open. We examine differences between firm-managed and customer-managed brand communities regarding the impact of perceived psychographic homogeneity, availability of virtual avenues and relationship with the brand on the community's influence on members and the assessments and intentions of community participants. Data were obtained from 555 respondents in two leading Microsoft XBOX brand communities in Brazil. Results indicate that management of the community of origin is the moderator of all considered relationships. Also, the most favorable effects for the company occur in the community that is not directly controlled and managed by the company itself. Brand loyalty, however, is higher for members of the official brand community. Guidelines on how companies can benefit from consumer-managed communities are discussed.

Keywords Consumer behavior, virtual communities, brand communities, brands, marketing strategy.

Resumen Los beneficios de las empresas al mantener comunidades virtuales de marca propia versus los provenientes de las comunidades creadas por los consumidores permanecen desconocidos. Este estudio prueba el efecto moderador del tipo de gestión de la comunidad en el impacto de la homogeneidad sicográfica percibida, de la disponibilidad de avenidas virtuales y de la relación con la marca sobre las influencias de la comunidad y las intenciones de los participantes de la comunidad. Los datos provienen de 555 participantes activos de dos grandes comunidades virtuales para el game XBOX en Brasil. Los resultados comprueban que la gestión de la comunidad de origen es moderadora de todas las relaciones consideradas. Apuntan también que algunos de los efectos más favorables para la empresa ocurren en la comunidad gestionada por los consumidores. La lealtad a la marca, no obstante, es mayor entre miembros de la comunidad oficial. El estudio indica cómo las empresas pueden beneficiarse con las comunidades gestionadas por consumidores.

Palabras clave Comportamiento del consumidor, comunidades virtuales, comunidades de marca, marca, estrategia de marketing.
\end{abstract}




\section{INTRODUÇÃO}

As comunidades virtuais de marca são definidas na literatura como "uma comunidade de ligação especializada e não definida geograficamente, baseada em um conjunto estruturado de relações sociais entre os admiradores de uma marca" (MUÑIZ e O'GUINN, 2001, p. 412). Essas comunidades estão ganhando maior importância, não apenas como centros condutores de informação, locais de suporte social e canais de autoexpressão, mas também, principalmente, como programas de marketing suportados direta ou indiretamente pelas empresas (ALGESHEIMER e DHOLAKIA, 2006; LI e BERNOFF, 2008; MCALEXANDER e outros, 2002).

Uma consequência desse interesse crescente é que as marcas que permitem e obtêm grande envolvimento do consumidor muitas vezes possuem várias comunidades de marca estabelecidas. Algumas dessas comunidades são organizadas e gerenciadas pelos profissionais de marketing das próprias empresas, outras são fundadas e geridas independentemente pelos entusiastas dessas marcas (DHOLAKIA e outros, 2009; MUÑIZ e O'GUINN, 2001; MUÑIZ e SCHAU, 2005; PORTER e DONTHU, 2008; WEBER, 2007).

Estudos recentes (MATHWICK e outros, 2008; PORTER e DONTHU, 2008) têm pontuado que há dois importantes direcionadores para o sucesso das comunidades virtuais de marca: (1) ter os participantes corretos na comunidade e (2) encorajá-los a interagir uns com os outros de maneira amistosa e prestativa por meio da disponibilização das corretas avenidas para tanto. Questões como essas são igualmente pertinentes para comunidades de marca gerenciadas pelos consumidores ou pela própria organização, ainda que diferenças fundamentais entre essas comunidades devam ser compreendidas e exploradas.

Apesar do número de trabalhos que têm se dedicado a pesquisar comunidades de marca virtuais nos últimos anos, mostrando a sua relevância teórica e econômica, estudos anteriores sobre comunidades de marca pouco se detiveram sobre as diferenças entre comunidades gerenciadas pelos consumidores e pela própria organização. Essa carência de estudos fez com que Porter e Donthu (2005) sugerissem a aplicação de pesquisas em comunidades de marca não suportadas pelas empresas e Algesheimer e outros (2005) discutissem se as comunidades organizadas por consumidores entusiastas poderiam ser menos susceptíveis aos efeitos negativos que podem ocorrer a empresas que gerenciam suas próprias comunidades. Os autores também encorajam futuros estudos sobre o tema que busquem compreender se realmente é mais interessante para as empresas gerenciarem as suas comunidades ou simplesmente deixá-las florescer por iniciativa dos consumidores.

Buscando investigar essa lacuna no estado da arte sobre o tema, este trabalho busca verificar o efeito moderador da comunidade de origem sobre as relações propostas em um framework teórico acerca do comportamento de consumidores participantes em comunidades virtuais de marca. O presente estudo sugere que as relações teóricas verificadas no framework proposto sejam afetadas, ou seja, tenham intensidades diferentes, dependendo do tipo de gestão da comunidade virtual de marca, seja gerenciada pela empresa ou pelos próprios consumidores. As comunidades pesquisadas eram da marca XBOX da Microsoft no Brasil.

Nosso fim aqui não é apenas compreender similaridades e diferenças entre comunidades gerenciadas pelos consumidores ou pelas empresas, mas prover insights específicos para os gestores das comunidades quando tomadores de decisões relativas à composição ou gestão de comunidades virtuais de marca.

\section{CONSTRUCTOS DE INTERESSE E HIPÓTESES DE PESQUISA}

Entre os diversos conceitos relevantes para o conhecimento de comunidades de marca, um dos primeiros a serem estudados refere-se à homogeneidade entre grupos. Esse conceito foi primeiramente abordado por meio das teorias sobre similaridade grupal, com forte embasamento na teoria da identidade social (DITOMASO e outros, 2007; ZELLMER-BRUHN e outros, 2008). Nesse contexto, o conceito de similaridade percebida é definido por Zellmer-Bruhn e outros (2008, p. 41) como "o grau em que os membros veem a eles mesmos como tendo poucas diferenças".

A distinção entre homogeneidade demográfica e psicográfica é importante no estudo das comunidades de marca por, pelo menos, duas razões. Primeiramente porque estas são variáveis de segmentação e posicionamento usadas para escolher participantes potenciais das comunidades por seus administradores. Os próprios participantes estão cientes dessas diferenças, uma vez que são visíveis nas interações off-line e aparecem frequentemente nas conversações que ocorrem nas interações on-line, assim como podem ser deduzidas com 
base no perfil postado pelos membros. Em segundo lugar, esses dois tipos de diferenças são teoricamente distintos, uma vez que a psicologia organizacional distingue os conceitos de homogeneidade demográfica, baseada em atributos realmente detectáveis, que podem ser rápida e consensualmente determinados após uma exposição relativamente breve da pessoa-alvo, e homogeneidade psicográfica, que é baseada em atributos latentes, que estão mais sujeitos a construção e são mais mutáveis (DITOMASO e outros, 2007; ZELLMER-BRUHN e outros, 2008). A homogeneidade psicográfica é nosso foco justamente por essa razão.

Dholakia e outros (2009) definem homogeneidade psicográfica percebida (HPP) como percepções dos participantes da extensão em que outros membros são iguais a eles em seus valores, interesses e hobbies. Consumidores com maiores níveis de homogeneidade ou similaridade também terão mais desejo em compartilhar seus interesses comuns pela marca, participando mais das atividades virtuais da comunidade, aumentando a sua identificação com a comunidade e a participação nessa comunidade.

Embora comunidades gerenciadas pelos consumidores e administradas pela organização tenham características comuns, como serem formadas por clientes que são fãs da marca e estão interessados nos mesmos assuntos, notícias e informações sobre a marca e seus competidores, estas têm também uma série de diferenças significativas. Talvez a mais importante destas distinções recaia sobre as razões do gestor da comunidade. As empresas criam comunidades de marca para cumprir objetivos de marketing (LI e BERNOFF, 2008; WEBER, 2007). Algumas também usam as comunidades de marca para ter insights por meio do monitoramento das discussões e/ou interação com os participantes. Outras o fazem para aumentar a lealdade dos participantes a seus produtos e marcas (MCALEXANDER e outros, 2002), aumentando o comportamento de compra e recompra (ALGESHEIMER e DHOLAKIA, 2006). Por fim, ainda que os próprios consumidores decidam a que comunidade unirem-se, há pelo menos algum grau de segmentação por parte dos gestores das comunidades, e, em se tratando de comunidades oficiais da marca, estas são parte do plano e política de marketing da empresa. Não é incomum para as empresas usarem variáveis conhecidas de segmentação, como aquelas com o foco demográfico ou psicográfico para recrutar participantes (WEBER, 2007).

As comunidades gerenciadas pelos consumidores, por outro lado, raramente especificam objetivos de marketing. Entretanto, algumas vezes, essas comunidades criam uma aglomeração de pessoas com um perfil distinto daquele que é o foco da comunidade suportada pela empresa. Os participantes elegem a comunidade e filiam-se a ela por compartilharem a mesma paixão pela marca, o que geralmente também resulta em valores, hobbies, interesses e estilos de vida compartilhados (MUÑIZ e SCHAU, 2005). Essas diferenças sugerem que os consumidores em comunidades gerenciadas por seus pares devem ser mais similares em características psicográficas, afetando as relações subsequentes desse constructo. Estabelece-se a primeira hipótese composta:

H1a: o tipo de gestão da comunidade modera a relação entre os constructos homogeneidade psicográfica percebida e participação na comunidade.

H1b: o tipo de gestão da comunidade modera a relação entre os construtos homogeneidade psicográfica percebida e identificação com a comunidade.

Para que essa participação aqui proposta efetivamente ocorra, entretanto, os participantes devem estar motivados para tomar parte de uma comunidade de marca e ajudar os outros (MUÑIZ e SCHAU, 2005), sendo que a ligação do consumidor com a comunidade virtual se dá por meio de três elementos: interatividade, infraestrutura técnica e valor do serviço. O valor do serviço é entendido como o potencial da comunidade para entregar soluções aos seus membros, enquanto a interatividade é o que permite o fluxo e as trocas entre as partes envolvidas na comunidade. Assim, define-se para este estudo que a disponibilidade de avenidas virtuais (DAV) refere-se à infraestrutura técnica apresentada pelas comunidades. Essa infraestrutura é responsável pelas avenidas de alta e de baixa interatividade para comunicação entre os seus membros (BAGOZZI e outros, 2007), permitindo uma maior interatividade e a consequente entrega de valor. Listas de e-mails, quadros e grupos de notícias são exemplos de avenidas virtuais de baixa interatividade. Ao contrário, salas de bate-papo, jogos virtuais e domínios múltiplos são exemplos de avenidas virtuais de alta interatividade.

Quanto maior o número de avenidas virtuais que a comunidade tem para interação entre seus membros, e entre os membros e a empresa, maior a possibilidade de intensificar o fluxo de informações e a consequente solução de problemas. De uma forma mais geral, a maior disponibilidade de avenidas virtuais para comunicação que a comunidade compartilha com seus membros 
também influencia a identificação com a comunidade e a participação nessa comunidade.

Porém, segundo Achrol e Kotler (1999), uma das questões a serem discutidas com relação a comunidades de marca gerenciadas pelos consumidores refere-se a sua manutenção, uma vez que esta requer considerável esforço e recursos. Esses recursos devem vir do empenho e doações dos membros, uma vez que não há verbas de marketing da organização para a sua manutenção. O que se espera é que as comunidades gerenciadas pelos consumidores tenham menos recursos para interatividade virtual de seus membros, traduzindo-se em um menor número de avenidas virtuais de alta e baixa interatividade (BAGOZZI e outros, 2007).

Resultados da fase netnográfica deste trabalho também apontam nesse sentido, revelando que as comunidades gerenciadas pelas empresas são mais organizadas do que as comunidades gerenciadas pelos consumidores e que as primeiras contêm maior disponibilidade de avenidas de comunicação virtual como salas de bate-papo, blogs, fóruns, quadros de notícias, fotos, músicas e outros. Os consumidores que participam das comunidades gerenciadas por outros consumidores sentem falta desses recursos que as comunidades gerenciadas pelas empresas possuem, por contarem com verbas de marketing das organizações. As comunidades gerenciadas pelas empresas dispõem de recursos para manter funcionários moderando e interagindo em salas de bate-papo ou respondendo às questões deixadas em quadros de notícias. Baseada nesses argumentos, se estabelece a segunda hipótese composta:

H2a: o tipo de gestão da comunidade modera a relação entre os constructos disponibilidade de avenidas virtuais e participação na comunidade.

H2b: o tipo de gestão da comunidade modera a relação entre os constructos disponibilidade de avenidas virtuais e identificação com a comunidade.

Algesheimer e outros (2005, p. 23) definem qualidade do relacionamento com a marca (RCM) como "o grau em que o consumidor vê a marca como um parceiro satisfatório em um relacionamento com ela". Essa definição será a adotada neste trabalho e inclui, de um lado, elementos cognitivos, como a crença do consumidor de que a imagem da marca é congruente com a sua autoimagem e a informação transmitida aos outros por meio do uso da marca, e, de outro, elementos emocionais, como o grau da ligação emocional dos consumidores com a marca. Segundo os autores, o relacionamento com a marca, para consumidores experientes e que já têm uma relação positiva com esta, é um antecedente da afiliação e participação do consumidor na comunidade de marca, sendo, então, o relacionamento com a marca precursor da identificação com a comunidade de marca. É presumível, porém, que o grau de relacionamento com a marca possa gerar diferentes níveis de identificação com a comunidade de marca, dependendo do tipo de gestão da comunidade, seja gerenciada pela empresa ou pelos próprios consumidores. Define-se a terceira hipótese:

H3: o tipo de gestão da comunidade modera a relação entre os constructos relacionamento com a marca e identificação com a comunidade de marca.

A identificação com a comunidade (ICC) é descrita por Algesheimer e outros (2005, p. 20) como "o quão uma pessoa constrói a si mesma como um membro, ou seja, como pertencente a uma dada comunidade de marca". Segundo os autores, essa identificação é coletiva ou compartilhada, e não uma identidade individual. O conceito de identificação com a comunidade é ancorado na identidade social, envolvendo seus componentes cognitivo, afetivo e avaliativo.

Com relação ao componente cognitivo, a identificação com a comunidade está relacionada ao processo de autocategorização, que faz com que o consumidor tenha ciência de pertencer ao grupo, enfatizando as similaridades com os membros e as dissimilaridades com os não membros. Conforme Algesheimer e outros (2005), essa dimensão capturaria a consciência compartilhada ou de pertença, descrita por Muñiz e O'Guinn (2001) como necessária para que uma coletividade possa ser considerada uma comunidade. Em uma comunidade virtual, a consciência da pertença ou participação ocorre quando o indivíduo crê que os seus valores vão ao encontro daqueles dos demais membros do grupo, fazendo com que as intenções conjuntas de seguir participando na comunidade sejam maiores (BAGOZZI e DHOLAKIA, 2002).

No que tange ao componente afetivo, a identificação com a comunidade implica um senso de envolvimento emocional com o grupo. Essa identificação promove o bem-estar do consumidor (ALGESHEIMER e outros, 2005) e significa que este concorda com as normas, tradições, rituais e objetivos da comunidade, suas características fundamentais formadoras descritas por Muñiz e O'Guinn (2001). O componente avaliativo da identidade social estaria, então, presente por meio 
da formação da autoestima por conta da afiliação à comunidade, implicando que o participante valoriza a sua identidade como membro da comunidade (ALGESHEIMER e outros, 2005). Pesquisas mostram que indivíduos que se identificam com um grupo em particular tendem a imitar seus membros para reforçar a sua identidade (MACKIE, 1986) e uma maior identificação com a comunidade leva a maiores influências da comunidade por conta dessa identificação. Essa relação, porém, dependerá do tipo de gestão da comunidade de consumidores, levando à seguinte hipótese de pesquisa:

H4: o tipo de gestão da comunidade modera a relação entre os constructos identificação com a comunidade e influências da comunidade.

A participação na comunidade (PNC), outro fator com potencial efeito sobre o comportamento do usuário, refere-se à interatividade deste na comunidade. Burnett (2000) divide o comportamento dos membros em não interativos - aqueles que apenas leem, não deixando mensagens, caracterizando um comportamento de busca de informações - e interativos - aqueles que geram conteúdo. Essa classificação é similar a de Algesheimer e Dholakia (2006) em "entusiastas" ou "indivíduos que obtêm benefícios da interação social sem contribuir".

Em comunidades de marca, também é muito comum que os administradores confiram status aos membros, baseados na sua contribuição para a comunidade (DHOLAKIA e outros, 2009; MATHWICK e outros, 2008). Os participantes usam seu status para influenciar o funcionamento da comunidade, assim como os outros membros (ALGESHEIMER e outros, 2005). O que se conclui é que consumidores que participam mais ativamente na comunidade, respondendo às questões de outros membros, participando em eventos face a face e deixando perguntas em salas de bate-papo etc., são aqueles que têm maior tendência a participação, afiliação e recomendação (ALGESHEIMER e outros, 2005), o que gera uma maior identificação com essa comunidade. Pesquisas anteriores também mostraram que a identificação com a comunidade de marca é relacionada às interações dos participantes uns com os outros e com a comunidade e ao valor que deriva dessas interações (DHOLAKIA e outros, 2009). Esses resultados revelam que quanto maior a participação, maior a identificação do consumidor com seus pares e maiores as influências desses pares sobre o comportamento do consumidor, o que leva ao estreitamento dos laços e relacionamentos estabelecidos e impacta o prolongamento da participação (NAMBISAN e BARON, 2007). Considerando-se comunidades de marca com diferentes perfis de gestão, espera-se que essas relações tenham intensidades diferentes. Portanto, definem-se como hipóteses:

\footnotetext{
H5a: o tipo de gestão da comunidade modera a relação entre os constructos participação na comunidade e identificação com a comunidade.

H5b: o tipo de gestão da comunidade modera a relação entre os constructos participação na comunidade e influências da comunidade.
}

As influências da comunidade (InC) são definidas aqui como o impacto das opiniões da comunidade nas opiniões dos membros e decisões sobre a categoria de produto e a marca (DHOLAKIA e outros, 2009). Um grande número de estudos sobre comunidades de marca têm mostrado que as comunidades exercem influência substancial nos seus membros e impactam seus pensamentos e ações (ex.: ALGESHEIMER e outros, 2005; MUÑIZ e HAMER, 2001). Bagozzi e Dholakia (2006a) concluíram que maiores intenções compartilhadas entre os membros da comunidade levam a maiores comportamentos relacionados ao produto em análise, como gasto de dinheiro com o produto e uso da marca. Dessa forma, consumidores que pretendem continuar a ter um relacionamento com a marca e com a comunidade terão uma maior tendência a mostrar mais comportamentos relacionados ao mercado, como comportamento de recompra da marca (ALGESHEIMER e outros, 2005), assim como maior ligação emocional com a marca (ALGESHEIMER e DHOLAKIA, 2006). O que se espera, portanto, é que as influências que a comunidade tem nas decisões de compra do consumidor para com a categoria de produto e a marca afetem positivamente as chamadas avaliações e intenções.

As avaliações e intenções (A\&I) que se dão em função das influências da comunidade são expressas neste estudo pelas intenções em ser leal à marca, em recomprar a marca e em agir como advogado de defesa da marca. Quanto mais integrado estiver o consumidor na comunidade de marca, mais leal ele é ao consumo dessa marca, pois maior é a identificação com a marca que os consumidores consomem como indivíduos e também como parte da comunidade em si (MCALEXANDER e outros, 2002). Conforme Bagozzi e Dholakia (2006b), a formação de relacionamentos com consumidores que têm os mesmos interesses na marca 
é uma maneira confiável e de impacto de persuadir e ligar os consumidores com ela, levando-os a ter maiores comportamentos associados à recompra e à lealdade (BENNET e RUNDLE-THIELE, 2005). Entende-se que as avaliações e intenções serão diversas quando o consumidor estiver ou não exposto a uma comunidade que seja oficialmente gerida pela marca em questão. Apresenta-se a última hipótese deste estudo:

H6: o tipo de gestão da comunidade modera a relação entre os constructos influências da comunidade e avaliações e intenções.

Graficamente, podem-se expressar as relações teóricas estabelecidas pelo estudo na Figura 1 abaixo.

\section{MÉTODO DE ESTUDO}

Como método para desenvolvimento do framework teórico e questionário de pesquisa, foi realizado um estudo exploratório preliminar, por meio de uma revisão da literatura e de uma pesquisa netnográfica em seis diferentes comunidades para dois diferentes produtos/marcas: LabVIEW, da National Instruments, e XBOX, da Microsoft. A análise do conteúdo das ob- servações levantadas nessa coleta foi feita conforme as premissas dadas por Kozinets (2002), analisando-se primeiramente individualmente cada comunidade e, posteriormente, grupos de achados comuns nas comunidades observadas, sendo os principais achados relativos aos seguintes tópicos: (1) à fidelidade dos consumidores às marcas; (2) à liberdade para interações e disponibilidade de avenidas para tanto; (3) ao foco, por determinados tipos de comunidades, em grupos-alvo específicos e bem definidos; (4) ao papel das organizações nas comunidades, inclusive naquelas gerenciadas pelos próprios consumidores.

A escolha da marca XBOX para o estudo quantitativo foi feita em função de ter comunidades com expressivo número de participantes, de ter como língua oficial o português e de já ter sido pesquisada na etapa qualitativa. As comunidades utilizadas na fase quantitativa foram a comunidade oficial da marca no País - XBOX Brasil (http://www.xbox.com/pt-BR) - e a maior comunidade gerenciada pelos consumidores no Brasil - Portalxbox (http://www.portalxbox.com. br). O questionário de pesquisa buscava mensurar crenças, atitudes e comportamentos com respeito à comunidade de origem, aos produtos XBOX e à Microsoft. Foram acrescidos, ainda, outros três blocos de questões de caracterização do respondente: (1) caracterização de uso da internet; (2) caracterização de uso

\section{Figura 1 - Framework proposto}

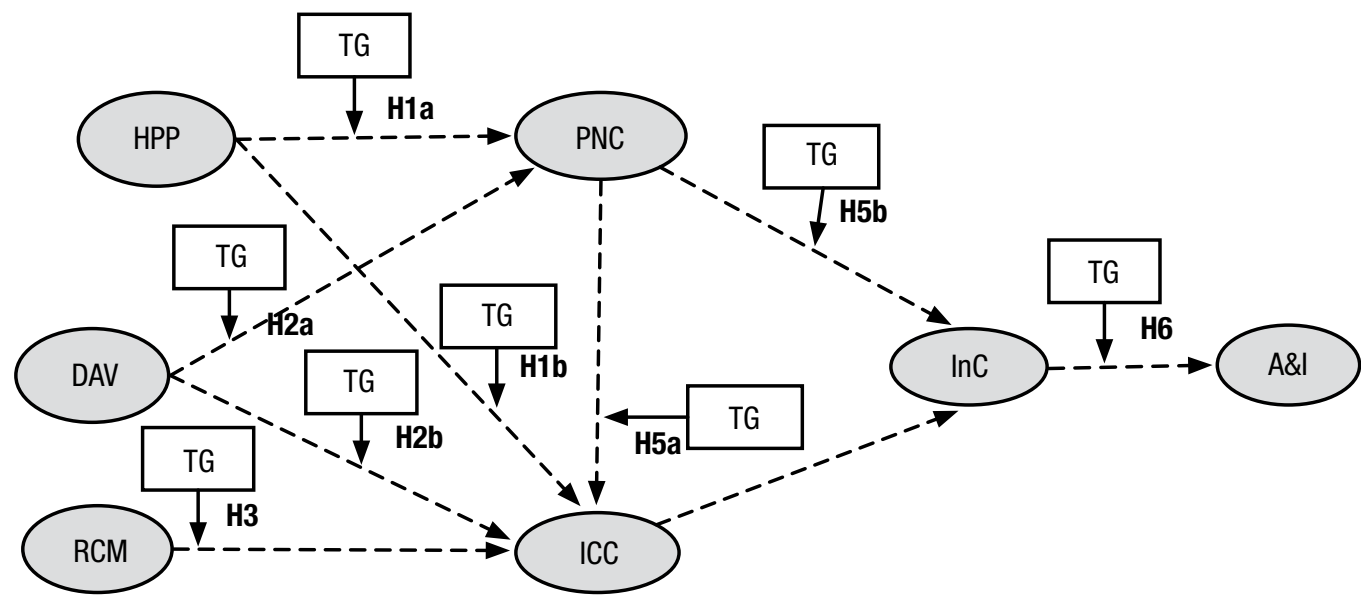

Legenda: HPP: homogeneidade psicográfica percebida; DAV: disponibilidade de avenidas virtuais; RCM: relacionamento com a marca; PNC: participação na comunidade; ICC: identificação com a comunidade; InC: influências da comunidade; A\&l: avaliações e intenções; TG: tipo de gestão.

Setas tracejadas $(--\rightarrow$ indicam hipóteses testadas em estudos prévios, setas cheias (

significam hipóteses investigadas neste trabalho. 
e participação nas comunidades de marca XBOX; (3) perfil do respondente. Utilizou-se, para mensuração das variáveis ligadas a cada construto do framework teórico (vide Quadro 1), uma escala do tipo Likert de concordância com 7 pontos.

O instrumento de coleta foi revisado e validado por quatro gestores de comunidades de marca e dois experts internacionais sobre o assunto. Posteriormente, o questionário de pesquisa foi disponibilizado aos membros das comunidades nas suas respectivas plataformas virtuais por meio de um link para "Pesquisa" que levava o respondente diretamente à página do questionário. Previamente, os pesquisadores postaram mensagens em ambas as comunidades explicitando os objetivos do estudo e solicitando a colaboração. A amostra alcançada foi de 555 casos, sendo 336 da comunidade Portalxbox e 219 da comunidade XBOX Brasil.

O processamento dos dados foi realizado utilizando-se os softwares SPSS 15.0 e LISREL 8.5. Seguiu-se o procedimento de processamento de estatísticas descritivas, purificação das escalas de medidas dos constructos, validação dos constructos propostos - por meio da

\section{Quadro 1 - Variáveis latentes e variáveis observáveis}

\section{Homogeneidade Psicográfica Percebida - HPP}

(v1) Os outros membros da [nome da comunidade] e eu temos os mesmos valores; (v2) Eu acho que os membros da [nome da comunidade] são muito similares quanto a seus interesses e hobbies; (v3) Eu tenho a mesma visão de mundo que a maioria dos membros da [nome da comunidade].

\section{Disponibilidade de Avenidas Virtuais - DAV}

(v4) A [nome da comunidade] tem muitas avenidas virtuais de alta interatividade, tais como salas de bate-papo, para seus membros se comunicarem uns com os outros; (v5) A [nome da comunidade] tem muitas avenidas virtuais de menor interatividade, tais como listas de e-mail, fóruns e grupos de correspondência para seus membros se comunicarem uns com os outros; (v6) A [nome da comunidade] tem muitas opções de avenidas virtuais; (v7) Eu consigo achar um local adequado ao meu estilo de comunicação na [nome da comunidade].

\section{Identificação com a Comunidade - ICC}

(v8) Eu me vejo como parte da [nome da comunidade]; (v9) Eu tenho encontrado pessoas maravilhosas devido a esta comunidade de marca; (v10) A imagem que faço de mim coincide perfeitamente com a identidade da [nome da comunidade].

\section{Relacionamento com a Marca - RCM}

(v11) Eu me sinto emocionalmente ligado(a) à marca XBOX; (v12) A marca XBOX tem um papel importante na minha vida; (v13) A imagem da marca XBOX e a minha própria imagem são similares em muitos aspectos; (v14) Eu sinto um forte senso de identificação com esta marca; (v15) Esta marca diz muito sobre o tipo de pessoa que eu sou.

\section{Participação na Comunidade - PNC}

(v16) Eu ajudo outros membros da [nome da comunidade] respondendo a suas perguntas e dúvidas; (v17) Eu passo muito tempo toda semana me comunicando com outros na [nome da comunidade]; (v18) Eu sou motivado(a) a participar em várias atividades da [nome da comunidade]; (v19) Eu gosto de dar apoio a outros membros da [nome da comunidade]; (v20) Eu usualmente contribuo para gerar um fluxo de comunicação interessante entre os membros da comunidade.

\section{Influências da Comunidade - InC}

(v21) A [nome da comunidade] tem muita influência com relação às minhas decisões de compra sobre videogames e jogos on-line; (v22) A [nome da comunidade] tem muita influência nas minhas opiniões sobre a marca XBOX; (v23) Após começar a participar da comunidade, eu compro mais produtos Microsoft; (v24) Eu vou continuar a comprar produtos Microsoft por causa do meu envolvimento com a [nome da comunidade].

\section{Avaliações e Intenções - A\&l}

(v25) Eu estou bastante satisfeito(a) com a [nome da comunidade]; (v26) Eu estou bastante satisfeito(a) com o XBOX; (v27) Eu estou bastante satisfeito(a) com a Microsoft; (v28) Eu falo coisas positivas sobre a Microsoft aos meus amigos e parentes; (v29) Eu recomendarei a Microsoft a qualquer um que solicitar o meu conselho; (v30) Eu pretendo continuar a comprar produtos Microsoft no futuro; (v31) Eu continuarei a ser um(a) cliente leal da Microsoft. 
análise fatorial confirmatória - e teste do modelo teórico proposto, utilizando-se a modelagem de equações estruturais (JÖRESKOG e SÖRBOM, 1996).

Posteriormente à validação do modelo estrutural, foi realizada uma análise multigrupos. Conforme Sauer e Dick (1993), os efeitos das variáveis moderadoras podem ser testados por meio da modelagem de equações estruturais, utilizando-se o moderador para dividir a amostra em grupos. Valeu-se, posteriormente, do teste do qui-quadrado para verificar a significância da diferença entre os parâmetros estruturais entre os grupos. Os procedimentos estatísticos devem testar e medir o efeito da variável independente na variável dependente como função do moderador (BARON e KENNY, 1986). Como variável moderadora, neste estudo, foi utilizada a comunidade de origem. Mais especificações sobre o teste multigrupos serão apresentados juntamente com seus respectivos resultados.

\section{RESULTADOS ALCANÇADOS}

Em função de o framework apresentado neste estudo já ter sido testado e validado em estudo anterior (ALMEIDA e outros, 2011), este trabalho presta-se somente a apresentar resumidamente os resultados dessa validação, tendo como objetivo maior a análise de moderação.

Considerando-se o teste do modelo estrutural, foi identificado apenas um forte índice de modificação entre as variáveis latentes do modelo, sugerindo a inserção de um vínculo entre os constructos relacionamento com a marca e avaliações e intenções (RCMàA\&I - índice 88,39). Ainda que o modelo tenha apresentado um bom ajuste sem a inserção desse índice de modificação, considerou-se a relevância teórica dessa proposição, optando-se por sua inserção. As medidas de ajustamento para o modelo contendo as duas amostras ficaram: Qui-Quadrado ( $\mathrm{c}^{2}$ ): 1032,31; Graus de Liberdade (gl): 311; $\mathrm{c}^{2} / \mathrm{gl}: 3,319$; $p$ : 0,000; GFI: 0,88; AGFI: 0,85; NNFI: 0,92; CFI: 0,93; RMSEA: 0,065. Os índices atingiram níveis considerados muito bons, com exceção dos valores do GFI e AGFI, que ficaram um pouco abaixo do valor de 0,9, comumente aceito. Entretanto, podem ser considerados como uma adequação satisfatória na medida em que sejam aceitos valores mais flexíveis referentes aos índices de ajustamento (por exemplo, aceitação de GFI e AGFI > 0,85) (MARSH, HAU, WEN, 2004). Embora o c2 seja significante, isso ocorre em amostras grandes como a que se apresenta neste estudo, não invalidando o ajustamento do modelo.

Os testes efetuados mostraram que as hipóteses de mediação formuladas, considerando a amostra total de 555 usuários, foram suportadas com um nível de significância entre $p<0,001$ e $p<0,05$, incluindo a nova relação proposta, exceção feita à relação entre participação na comunidade e influências da comunidade $(b=-0,002, n s)$, que será mais bem considerada na análise multigrupos.

\section{Os efeitos das variáveis moderadoras - análise multigrupos}

Para a análise multigrupos - foco central deste estudo -, foi seguido o procedimento proposto por Sauer e Dick (1993) no qual duas rodadas de análise de dados são requeridas. Na primeira rodada, os parâmetros estruturais são restritos, para serem iguais entre os grupos. Esse procedimento gera uma matriz de covariância estimada para cada grupo e um valor de qui-quadrado para o grupo de modelos, em que o qui-quadrado representa a medida de ajuste geral para todos os modelos de todos os grupos (JÖRESKOG e SÖRBOM, 1996). Na segunda rodada, as restrições dos parâmetros estruturais são removidas, resultando em um segundo valor de qui-quadrado. Aqui, os diferentes grupos são especificados com todos os parâmetros livres para serem únicos para cada grupo. A existência ou não de efeito moderador é então verificada, fazendo-se a diferença entre os valores do qui-quadrado e o teste da hipótese nula. Posteriormente, buscando verificar-se o efeito moderador de cada relação estrutural do modelo, procede-se à restrição de uma relação por vez, seguida do teste do qui-quadrado. Ambos os procedimentos foram empregados, e os resultados podem ser conferidos na Tabela 1, que aponta ser a comunidade de origem moderadora de todas as relações sugeridas para o modelo. Posteriormente, a análise do vetor Kappa permitiu verificar diferenças nas médias das variáveis latentes entre os grupos, reforçando a conclusão extraída.

Por meio da análise das estatísticas dos parâmetros de ligação para cada uma das relações do modelo, que apresentou diferenças significativas entre os grupos, pode-se perceber que, para a comunidade XBOX Brasil, há uma relação significativamente maior entre a homogeneidade psicográfica percebida e a identificação com a comunidade (HPPàICC $=0,24 ; p<0,01$, para XBOX Brasil e 0,19; $p<0,001$, para Portalxbox) 
e homogeneidade psicográfica percebida e o nível de participação na comunidade (HPPàPNC $=0,38 ; p<$ 0,001 , para XBOX Brasil e HPPàPNC $=0,27 ; p<0,001$, para Portal XBOX), suportando H1a e H1b.

A relação entre a disponibilidade de avenidas virtuais e o nível de participação na comunidade foi significante apenas para a comunidade XBOX Brasil (DAVàPNC $=0,25 ; p<0,01$, para XBOX Brasil e DAVàPNC $=0,12$, não significante, $\operatorname{com} p \approx 0,08$, para Portalxbox), sendo, portanto, aceita a hipótese H2a, uma vez que fica demonstrada a diferença significante dos valores. A relação do primeiro constructo com a identificação com a comunidade foi significativamente mais forte para a comunidade gerenciada pela Microsoft (DAVàICC = 0,26; $p<0,001$ para XBOX Brasil e DAVàICC $=0,15$; $p<0,01$ para Portalxbox), suportando, portanto, H2b.

A comunidade gerenciada pelos consumidores mostrou maior capacidade de gerar identificação com a comunidade com base no relacionamento com a marca do que as gerenciadas pelo usuário $(\mathrm{RCM} \rightarrow \mathrm{ICC}$ $=0,25 ; p<0,001$ para Portalxbox e RCM $\rightarrow$ ICC = 0,20; $p<0,001$ para XBOX Brasil), levando à aceitação de H3. Igualmente, a comunidade gerenciada pelos consumidores caracteriza-se por apresentar uma relação significativamente maior entre a identificação com a comunidade e as influências da comunidade
$($ ICC $\rightarrow$ InC $=0,94 ; p<0,001$ para Portalxbox e 0,$38 ; p$ $<0,001$ para XBOX Brasil), levando à aceitação da H4.

A relação entre a participação na comunidade e a identificação com a comunidade foi, também, significativamente maior para usuários do Portalxbox $(\mathrm{NPC} \rightarrow \mathrm{ICC}=0,69 ; p<0,001$ para Portalxbox e 0,$57 ; p$ $<0,001$ para XBOX Brasil). Verificou-se, ainda, a relação entre participação na comunidade e influências da comunidade, tendo, entretanto, valor negativo para a comunidade Portalxbox (NPC $\rightarrow \operatorname{InC}=-0,42 ; p<0,001)$ e positivo para a comunidade XBOX Brasil $(\mathrm{NPC} \rightarrow \mathrm{InC}=$ $0,40 ; p<0,001)$. Tais resultados antagônicos podem ter implicações no teste do modelo estrutural com a base conjunta e merecem discussão à parte. As evidências, porém, sustentam as hipóteses $\mathrm{H} 5 \mathrm{a}$ e $\mathrm{H} 5 \mathrm{~b}$. A relação entre as influências da comunidade e as avaliações e intenções mostrou-se não significante para usuários do Portalxbox (InCàA\&E $=1,52 ; n s$ para Portalxbox e InC $\rightarrow$ A\&E $=0,57 ; p<0,001$ para XBOX Brasil), implicando uma diferença significante entre os valores das relações e, portanto, levando à aceitação de H6.

\section{Análise do vetor Kappa}

Com o propósito de comparar os valores das variáveis latentes das duas comunidades de consumidores, em-

\section{Tabela 1 - Teste de qui-quadrado da análise multigrupos para comunidade de origem}

\begin{tabular}{|c|c|c|c|c|c|c|c|c|c|c|}
\hline Relação Fixa & $\chi^{2}$ & gl & $\Delta \chi^{2}$ & DGL & p & CFG & Estatistica $t$ & CFG & t-value & Interpretação \\
\hline $\begin{array}{l}\text { Nenhuma: } \\
\text { modelo livre }\end{array}$ & 1419,16 & 622 & & & & \multirow{2}{*}{\multicolumn{2}{|c|}{ Portalxbox }} & \multirow{2}{*}{\multicolumn{2}{|c|}{ XBOX Brasil }} & \\
\hline modelo restrito & 1939,95 & 632 & 520,8 & 10 & $<0,00$ & & & & & \\
\hline $\mathrm{TG} \rightarrow[\mathrm{HPP} \rightarrow \mathrm{ICC}]$ & 1433,04 & 623 & 13,88 & 1 & $<0,00$ & 0,19 & $3,42^{\star \star *}$ & 0,24 & $3,36^{\star \star}$ & H1a aceita \\
\hline $\mathrm{TG} \rightarrow[\mathrm{HPP} \rightarrow \mathrm{NPC}]$ & 1440,84 & 623 & 21,68 & 1 & $<0,00$ & 0,27 & $3,83^{\star * *}$ & 0,38 & $4,39^{\star \star \star}$ & H1b aceita \\
\hline $\mathrm{TG} \rightarrow[\mathrm{DAV} \rightarrow \mathrm{NPC}]$ & 1425,98 & 623 & 6,82 & 1 & $<0,01$ & 0,12 & $1,76 n s$ & 0,25 & $3,29^{\star *}$ & H2a aceita \\
\hline $\mathrm{TG} \rightarrow[\mathrm{DAV} \rightarrow \mathrm{ICC}]$ & 1438,38 & 623 & 19,22 & 1 & $<0,00$ & 0,15 & $2,89^{\star \star}$ & 0,26 & $3,80^{\star \star \star}$ & $\mathrm{H} 2 \mathrm{~b}$ aceita \\
\hline $\mathrm{TG} \rightarrow[\mathrm{RCM} \rightarrow \mathrm{ICC}]$ & 1437,33 & 623 & 18,17 & 1 & $<0,00$ & 0,25 & $5,42^{\star \star \star}$ & 0,20 & $3,41^{\star \star \star}$ & H3 aceita \\
\hline $\mathrm{TG} \rightarrow[\mathrm{ICC} \rightarrow \ln \mathrm{C}]$ & 1431,63 & 623 & 12,47 & 1 & $<0,00$ & 0,94 & $7,16^{\star \star \star}$ & 0,38 & $3,46^{\star \star \star}$ & H4 aceita \\
\hline $\mathrm{TG} \rightarrow[\mathrm{PNC} \rightarrow \mathrm{ICC}]$ & 1485,87 & 623 & 66,71 & 1 & $<0,00$ & 0,69 & $12,16^{\star \star \star}$ & 0,57 & $7,59^{\star \star \star}$ & H5a aceita \\
\hline $\mathrm{TG} \rightarrow[\mathrm{PNC} \rightarrow \ln \mathrm{C}]$ & 1429,94 & 623 & 10,78 & 1 & $<0,00$ & $-0,42$ & $-3,61^{\star \star \star}$ & 0,40 & $3,78^{\star \star \star}$ & H5b aceita \\
\hline $\mathrm{TG} \rightarrow[\mathrm{InC} \rightarrow \mathrm{A} \& \mid]$ & 1436,86 & 623 & 17,7 & 1 & $<0,00$ & 0,09 & $1,52 n s$ & 0,31 & $4,82^{\star \star \star}$ & H6 aceita \\
\hline
\end{tabular}

Obs.: ${ }^{*} p<0,05,{ }^{* \star} p<0,01,{ }^{* \star *} p<0,001$

Legenda: CFG: carga fatorial entre grupos; HPP: homogeneidade psicográfica percebida; DAV: disponibilidade de avenidas virtuais; RCM: relacionamento com a marca; PNC: participação na comunidade; ICC: identificação com a comunidade; InC: influências da comunidade; A\&l: avaliações e intenções; TG: tipo de gestão. 
pregou-se o teste do vetor Kappa. Esse vetor aponta diferenças nas médias das variáveis latentes entre as comunidades de origem. Na Tabela 2, abaixo, valores negativos para o Kappa indicam que o segundo grupo (XBOX Brasil) tem médias mais baixas, e valores positivos indicam que o referido grupo tem médias mais altas. Os t-values validam a significância estatística de tal diferença entre médias e os valores entre parênteses indicam a média aritmética das variáveis do constructo.

O exame das médias das variáveis latentes e dos valores do vetor Kappa permite que se observe que apenas para o constructo avaliações e intenções a média da comunidade gerenciada pela Microsoft, XBOX Brasil, é significantemente superior à média da comunidade gerenciada pelos consumidores, Portalxbox. Para todos os demais constructos analisados, à exceção de relacionamento com a marca, as médias da comunidade Portalxbox são maiores, ainda que, para a dimensão de homogeneidade psicográfica percebida, essa diferença não seja estatisticamente significativa.

\section{CONSIDERAÇÕES E IMPLICAÇÕES}

$\mathrm{Na}$ presente pesquisa, examinamos as diferenças entre comunidades virtuais gerenciadas pela organização e pelos consumidores. O teste do qui-quadrado para a análise multigrupos demonstrou ser a comunidade de origem moderadora das relações propostas no modelo estudado e haver diferenças significativas em todas as relações entre constructos desenhadas.

Acerca das relações hipotetizadas, um primeiro achado é que os gerentes de marketing podem considerar a composição de suas comunidades para fins de gestão e segmentação. A H1, acerca da homogeneidade psicográfica percebida, foi suportada e, ainda, verificou-se uma maior percepção dessa homogeneidade por parte dos participantes do Portalxbox. A comunidade gerenciada pela Microsoft, entretanto, mostrou-se mais eficiente em gerar identificação e participação na comunidade por conta da homogeneidade psicográfica percebida.

A composição da comunidade é determinada pela escolha dos consumidores ao unirem-se a ela (ALGESHIEMER e DHOLAKIA, 2006). Compreender por que esses consumidores escolhem a participação nas suas comunidades exige entender se as diferentes comunidades estão cumprindo objetivos diferentes, unindo consumidores com características demográficas ou psicográficas similares sob seu domínio. Segundo Li e Bernoff (2008) e Weber (2007), é muito comum que as empresas segmentem suas comunidades por critérios demográficos; em contraste, as comunidades gerenciadas pelos consumidores podem estar se formando por grupos com características psicográficas mais similares, mas demograficamente negligenciados, sendo mais homogêneos em valores, interesses e hobbies. As comunidades gerenciadas pelos consumidores podem expandir cobertura e escopo para uma base maior de fãs do produto e da marca do que a própria empresa foca em sua comunidade e podem ajudar a empresa a identificar esses consumidores não atendidos que estão interessados em suas marcas. Maiores pesquisas são necessárias para lançar luz a essas possibilidades.

Com relação à percepção de disponibilidade de avenidas virtuais, esta também foi significativamente mais alta para o grupo do Portalxbox. Entretanto, embora os membros da comunidade Portalxbox percebam que têm à sua disposição uma maior gama de avenidas para interação, é para os membros do XBOX Brasil

\section{Tabela 2 - Teste de diferenças entre médias nas comunidades de origem}

\begin{tabular}{|l|c|c|c|}
\hline \multicolumn{1}{|c|}{ Constructos } & Portal XBOX & XBOX Brasil & Estatística $\boldsymbol{t}$ \\
\hline Homogeneidade Psicográfica Percebida & $0 /(4,0982)$ & $-0,42 /(3,7137)$ & $-1,49 n s$ \\
\hline Disponibilidade de Avenidas Virtuais & $0 /(5,1786)$ & $-0,72 /(4,3059)$ & $-5,30^{\star \star \star}$ \\
\hline Relacionamento com a Marca & $0 /(4,2500)$ & $-0,06 /(4,2539)$ & $-0,28 n s$ \\
\hline Identificação com a Comunidade & $0 /(5,0764)$ & $-1,01 /(3,8584)$ & $-5,36^{\star \star *}$ \\
\hline Nível de Participação na Comunidade & $0 /(4,6369)$ & $-0,94 /(3,7553)$ & $-4,92^{\star \star \star}$ \\
\hline Influências da Comunidade & $0 /(4,8229)$ & $-0,95 /(3,8973)$ & $-4,61^{\star \star *}$ \\
\hline Avaliações e Intenções & $0 /(4,5244)$ & $0,36 /(5,0849)$ & $2,33^{*}$ \\
\hline
\end{tabular}

Observações: ${ }^{*} p<0,05,{ }^{* \star} p<0,01,{ }^{* \star \star} p<0,001 \quad n s=$ não significante 
que a percepção de disponibilidade de avenidas para interação mais afeta a participação e a identificação.

A comunidade Portalxbox tem um perfil diferente de muitas comunidades gerenciadas pelos consumidores. Os fundadores e administradores dessa comunidade mantêm uma administração altamente profissionalizada e uma enorme gama de avenidas para interatividade virtual, que incluem editoriais altamente profissionalizados, podcasts e revistas virtuais. A comunidade gerenciada pela Microsoft, por sua vez, era menor, mais nova e contava com um reduzido número de moderadores à época do estudo. Embora ambas as comunidades possuíssem as avenidas virtuais básicas de alta e baixa interatividade, a gama de outros serviços oferecidos pelo Portalxbox pode ter elevado a percepção de disponibilidade de avenidas para comunicação por parte dos seus usuários.

A ligação entre relacionamento com a marca e identificação com a comunidade mostrou-se mais forte para o grupo Portalxbox. Em uma relação não pressuposta no teste de hipóteses, por o vínculo entre os constructos ter sido inserido apenas no teste do modelo, como explicitado anteriormente, o mesmo relacionamento com a marca, entretanto, foi mais eficiente em criar avaliações e intenções na comunidade gerenciada pela Microsoft. Essa última relação tem sentido teórico-prático, uma vez que a comunidade oficial da marca é justamente aquela em que o relacionamento com a marca XBOX afeta as intenções de lealdade à marca-mãe, Microsoft, com maior força.

Com relação aos resultados complementares, o teste do vetor Kappa mostrou que os membros da comunidade Portalxbox identificam-se mais com ela, têm maior participação e percepção de influência da comunidade nas decisões de compra individuais com relação ao produto e à marca do que os membros da comunidade gerenciada pela Microsoft. Paralelamente, as relações PNC $\rightarrow$ ICC e ICC $\rightarrow$ InC foram significativamente maiores para membros do Portalxbox. Pesquisas anteriores já demonstraram que os consumidores estão cientes sobre as tentativas de persuasão dos profissionais de marketing, fazendo com que eles se identifiquem com as comunidades oficiais em menor grau do que com aquelas gerenciadas por outros consumidores (DHOLAKIA e outros, 2009).

Interessantemente, a relação entre participação na comunidade e influências da comunidade apresentou-se positiva para a comunidade XBOX Brasil e negativa para o Portalxbox. Esses resultados demonstram que um maior nível de participação leva à maior influência da comunidade nas decisões de compra para os membros da comunidade gerenciada pela Microsoft, entretanto, quando se trata dos membros da comunidade gerenciada pelos próprios consumidores, uma maior participação na comunidade leva a menores influências da comunidade nas decisões sobre o produto e a marca.

Esse resultado ligado à comunidade gerenciada pelos consumidores, embora inesperado, pode estar relacionado ao ganho de confiança desses consumidores por conta da sua participação na comunidade e ao seu aprendizado individual (DHOLAKIA e outros, 2009; MATHWICK e outros, 2008), de modo que quanto mais ele participa, maior a aprendizagem e menor a dependência da comunidade para decisões relativas ao produto e à marca. A aprendizagem individual, por sua vez, faz com que os consumidores passem a agir como funcionários parciais da empresa, facilitando a aprendizagem de outros membros.

A informação acurada e o design do site da comunidade também podem ter um papel fundamental em aumentar a aprendizagem do usuário (DHOLAKIA e outros, 2009). Em função de a comunidade gerenciada pelos consumidores ter uma maior percepção de disponibilidade de ferramentas de interatividade virtual, isso pode ter facilitado a aprendizagem do consumidor. Auxilia também nesse processo o fato de a comunidade gerenciada pelos consumidores ser mais antiga. A teoria que se propõe é que, nessas situações, quanto maior a participação na comunidade, menor a dependência desta para as decisões de compra sobre o produto e a marca, que são as influências da comunidade, em função de uma variável mediadora que poderia ter impacto nessa relação: a aprendizagem. Resultados do estudo de Algesheimer e outros (2010) já demonstram que os consumidores ficam mais seletivos nas compras quando fazem parte de comunidades. Estudos futuros são necessários, no entanto, para que essa hipótese possa ser comprovada.

A relação entre influências da comunidade e avaliações e intenções foi significativa apenas para o grupo XBOX Brasil. Esses dados justificam-se com base nas considerações tecidas anteriormente acerca da aprendizagem. Justificam-se, também, pelo fato de, efetivamente, os membros da comunidade gerenciada pela Microsoft estarem mais expostos às influências que essa comunidade, que é a comunidade oficial da marca, tem sobre as intenções de recomendação, recompra e lealdade relacionadas à própria Microsoft.

Os resultados do estudo trazem à luz um paradoxo interessante sobre as comunidades de marca, relacionado ao fato de que alguns dos efeitos mais favoráveis 
para a empresa ocorrem para a comunidade que não é diretamente controlada e administrada por ela. Comunidades de marca administradas por consumidores, como o Portalxbox, são grandes e bem-gerenciadas, além de serem formadas pelas motivações intrínsecas dos consumidores e seu alto grau de ligação com a marca (MUÑIZ e O'GUINN, 2001). Essas coletividades de consumidores são difíceis de replicar pelas empresas, especialmente quando vibrantes comunidades gerenciadas pelos consumidores já existem (BERTHON e outros, 2008). Se as comunidades oficiais são mais efetivas em gerar lealdade à marca-mãe ou empresa em suas diversas formas, as comunidades gerenciadas por consumidores são mais efetivas em gerar identificação com a comunidade e participação. Embora efetivamente a comunidade oficial gere mais efeitos de ponta de gôndola, os consumidores sentem-se mais conectados e participam mais nas comunidades gerenciadas pelos próprios.

As empresas devem atentar para o fato de que as diferentes comunidades podem estar cumprindo diferentes tarefas de marketing. Uma vez que os membros dessas comunidades têm interesses estritos, as empresas podem perfeitamente segmentar mercados para comunicação e mesmo vendas virtuais baseados nessas comunidades. Esse estudo oferece subsídios para gestores de marketing que estejam ambivalentes sobre os benefícios das comunidades gerenciadas pelos consumidores. As empresas podem, ainda, beneficiar-se das comunidades existentes, oferecendo encorajamento e suporte não financeiro para essas comunidades antes de decidir investir em uma comunidade gerenciada pela organização.

A despeito das contribuições do estudo, ele tem algumas limitações. Uma delas é o uso da metodologia survey, na qual as medidas foram coletadas em um único momento. Para corrigir essa limitação, uma série de procedimentos analíticos foi utilizada ainda na fase de validação dos constructos, como o estabelecimento de validade discriminante entre eles e a comparação do ajuste do modelo com modelos rivais. Outra é o uso de respondentes somente brasileiros e pertencentes a apenas duas comunidades para o mesmo produto e marca.

\section{REFERÊNCIAS}

ACHROL, R. S; KOTLER, P. Marketing in the network economy. Journal of Marketing, v. 63, Special Issue, p. 146163, 1999.
ALGESHEIMER, R; DHOLAKIA, U. M. The long-term effects of joining and participating in customer communities. In: EUROPEAN MARKETING ASSOCIATION, May, 2008, Provo. Anais. Provo: 37th EMAC Conference, Brighton, London, 2008.

ALGESHEIMER, R. e outros. The impact of customer community participation on customer behaviors: an empirical investigation. Marketing Science, v. 29, n. 4, p. 756-769, 2010.

ALGESHEIMER, R. e outros. The social influence of brand community: evidence from european car clubs. Journal of Marketing, v. 69, n. 3, p. 19-30, 2005.

ALMEIDA, S. O. e outros. Os efeitos da participação em comunidades virtuais de marca no comportamento do consumidor: proposição e teste de um modelo teórico. Revista de Administração Contemporânea, v. 15, n. 3, p. 366-391, 2011.

BAGOZZI, R. P; DHOLAKIA, U. M. Antecedents and purchase consequences of customer participation in small group brand communities. International Journal of Research in Marketing, v. 23, n. 1, p. 45-61, 2006b.

BAGOZZI, R. P; DHOLAKIA, U. M. Intentional social action in virtual communities. Journal of Interactive Marketing, v. 16, n. 2, p. 2-21, 2002.

BAGOZZI, R. P; DHOLAKIA, U. M. Open source software user communities: a study of participation in linux user groups. Management Science, v. 52, n. 7, p. 1099-1115, 2006a.

BAGOZZI, R. P. e outros. Antecedents and consequences of online social interactions. Media Psychology, v. 9, n. 1, p. 77-114, 2007.

BARON, R. M; KENNY, D. The moderator-mediator variable distinction in social psychological research: conceptual, strategic, and statistical considerations. Journal of Personality and Social Psychology, v. 51, n. 6, p. 1173-1182, 1986.

BENNET, R; RUNDLE-THIELE, S. The brand loyalty life cycle: implications for marketers. Journal of Brand Management, v. 12, n. 4, p. 250-263, 2005.

BERTHON, P; Pitt, L; Campbell, C. Ad lib: when customers create the ad. California Management Review, v. 50, n. 4 , p. 6-30, 2008. 
BURNETT, G. Information exchange in virtual communities: a typology. Information Research, v. 5, n. 4, p. 1-39, 2000.

DHOLAKIA, U. M. e outros. Communal service delivery: how customers benefit from participation in firm-hosted virtual P3 communities. Journal of Service Research, v. 12, n. 2, p. 208-226, 2009.

DITOMASO, N. e outros. Workforce diversity and inequality: power, status, and numbers. Annual Review of Socio$\log y$, v. 33, p. 473-501, 2007.

JÖRESKOG, K; SÖRBOM, D. LISREL 8: user's reference guide. Chicago: Scientific Software International, 1996. 378 p.

KOZINETS, R. V. The field behind the screen: using netnography for marketing research in online communities. Journal of Marketing Research, v. 39, n. 1, p. 61-72, 2002.

LI, C; BERNOFF, J. Groundswell: winning in a world transformed by social technologies. Boston: Harvard Business School Press, 2008. 224 p.

MACKIE, D. M. Social identification effects in group polarization. Journal of Personality and Social Psychology, v. 50, n. 4, p. 720-728, 1986.

MARSH, H; HAU, K; WEN, Z. In search of golden rules: comment on hypothesis-testing approaches to setting cutoof values for fit indexes and dangers in overgeneralizing Hu and Bentler's (1999) findings. Structural Equation Modeling, v. 11, n. 3, p. 320-341, 2004.

MATHWICK, C. e outros. Social capital production in a virtual P3 community. Journal of Consumer Research, v. 34, n.6, p. 832-849, 2008.

MCALEXANDER, J. H. e outros. Building brand community. Journal of Marketing, v. 66, n. 1, p. 38-54, 2002.

MUÑIZ, A. M. Jr; HAMER, L. O. Us versus them: oppositional brand loyalty and the cola wars. Advances in Consumer Research, v. 28, p. 355-361, 2001.

MUÑZ, A. M. Jr; O'GUINN, T. C. Brand community. Journal of Consumer Research, v. 27, n. 4, p. 412-432, 2001.

MUÑIZ, A. M. Jr; SCHAU, H. J. Religiosity in the abandoned apple Newton brand community. Journal of Consumer Research, v. 31, n. 4, p. 737-747, 2005.
NAMBISAN, S; BARON, R. Interactions in virtual customer environments: implications for product support and customer relationship management. Journal of Interactive Marketing, v. 21, n. 2, p. 42-62, 2007.

PORTER, C. E; DONTHU, N. Cultivating trust and harvesting value in virtual communities. Management Science, v. 54, n. 1, p. 113-128, 2008.

PORTER, C. E; DONTHU, N. Relationship marketing in virtual communities: the effect of a community sponsor's efforts to manage content, embeddedness and interaction on beliefs, trust and trust-based outcomes. [Unpublished working paper]. 2005.

SAUER, P. L; DICK, A. Using moderator variables in structural equation models. In: ADVANCES IN CONSUMER RESEARCH, 20, 1993, Provo. Anais. Provo: Association for Consumer Research, 1993.

WEBER, L. Marketing to the social web: how digital customer communities build your business. New Jersey: John Wiley \& Sons Inc, 2007.

ZELLMER-BRUHN, M. E. e outros. When do differences matter? An exploration of perceived similarity in teams. Organizational Behavior and Human Decision Processes, v. 107, n.1, p. 41-59, 2008. 